\title{
Influence of the Varnishing "Surface" Coverage on Optical Print Characteristics
}

\author{
Tomislav Hudika*, Igor Majnarić, Tomislav Cigula
}

\begin{abstract}
Varnishing is often used to protect or decorate the print, but it can also influence the print's colour appearance. Therefore, varnishing should enhance the print role, while colour change should be kept as low as possible. The aim of this paper is to evaluate inkjet UV LED varnishing by estimating optical print characteristics. Prepared offset prints were UV LED varnished in different surface coverage values (SCV) with a matte and gloss finish. The prints were evaluated by measuring gloss and colour coordinates. Secondly, varnishing was performed in a different SCV, which enabled assessing the optimal SCV, i.e. the most cost-efficient varnishing. The printing process was conducted in a standardized and controlled laboratory environment, regulated via the ISO 12647-2:2013 norm to ensure that the black colour was reproduced in the standard tolerance. The results showed that the colour difference is negligible (stays in the ISO tolerances), regardless of the varnishing SCV. On the other hand, the optimal printing gloss is at the SCV of $100 \%$.
\end{abstract}

Keywords: colour difference ( $\Delta \mathrm{E})$; inkjet; offset printing; print gloss; UV LED; varnishing

\section{INTRODUCTION}

The visual effect of segmental varnishing is becoming an increasingly desirable feature in the graphic industry. The initial usage of varnishing was to protect products. Today, almost every product, from commercial to personalized items, includes some type of varnishing. In terms of design, varnish is applied to the majority of products to increase their value by enhancing their visibility or to personalize the product for a customer. Varnishing could be, to some extent, conducted with most printing techniques, including screen printing, flexography, standard offset printing, drip-off offset systems and inkjet digital printing [1].

As the market leader in printing, offset printing is continuously developing so that it would remain at the top. The printing presses in offset printing have significantly improved their automatization, shortened the changeover time and various add-ons which enable customers to apply certain special effects, such as varnish, on the print in one go. On the other side, these highly productive printing presses include a higher price, workspace size and complexity, which is why other printing techniques are also developing.

One of the digital techniques which could be used for various applications is inkjet printing. Inkjet printing uses nozzles to spray ink in the form of a droplet which forms dots on the substrate's surface and therefore creates an image. Depending on the technological solution, it can print continuously or via "drop-on-demand", where it uses the UV curing technology (mercury vapor or LED).

Moreover, with inkjet, one can also print various varnish types, including the UV LED, in one or more layers, which gives the customer the possibility of relief on the print. When it comes to the market value, it is estimated that digital printing will grow from 21.35 Billion USD (2018) up to 33.49 Billion USD (2026). Hence, one must perceive it as a serious contender in the printing market $[2,3]$. One of the reasons for this growth is a potential hybrid, where inkjet is used to print the added value (printed electronics, various coatings) over the standard offset-printed image. Some researchers have already made some research with the UV
LED overprinting varnishes (OPVs) by printing varnish in a pattern, which resulted in a conclusion that gloss may influence the print colour and that the substrate surface has a great impact on the varnish finish in terms of gloss $[4,5]$. The purpose of this research is to determine the influence of the UV LED OPV on the offset prints that would be in accordance with the ISO 12647-2:2013 norm. Additionally, the research should give more insight on the relationship between the optical print characteristics and the varnishing tonal value.

\subsection{UV LED Inkjet Varnishing Technology}

Inkjet, as a printing technique, has the ability to print out layers by using only varnish. In the process of segmental UV LED varnishing by the means of inkjet printing machines, a thin transparent layer is formed on the surface of the desired substrate [6]. Varnishing could be done with a gloss and matt finish with the same varnish. The finishes are done by using a different light energy emission for curing. In the case of UV inks or varnishes, curing is performed by the photo-initiators' change under the UV light.

Varnish is composed of $10-20 \%$ acrylic amine synergist, $25-35 \%$ hexamethylene diacrylat hexane 1.6 diol diacrylate, $30-40 \%$ acrylic ester, $10-20 \%$ photosensitive monomers, 5$15 \%$ derivates based on phosphine oxides [7]. The principle of inkjet printing is the same for colour printing and varnishing, i.e. the ink or varnish droplet forms a layer on the printing substrate. The formation of printing areas is performed by ripping the image via FM screening and transferring a binary image onto the substrate [8].

Overprint varnishes (OPVs) are coatings applied over the already printed substrate for the reasons of protection, decoration, gloss enhancement, stain resistance, security purposes, resistance to discoloration, etc. The UV LED varnish is basically a UV LED ink without the pigments that has the possibility to change its finish by applying different light energy for curing. One can also do textured OPVs with the use of different patterns and layer thickness. OPVs can be used with any printing technique and by means of all curing 
types such as UV LED, oxypolimerization, air drying, IR curing etc. [9]. UV varnishes or inks do not evaporate or demand a high amount of solvents and due to these factors, they have great light, wear and temperature resistance to outer factors.

\section{EXPERIMENTAL}

The proposed research was conducted on samples prepared by combining two printing techniques, conventional offset printing with the printability tester and inkjet varnishing.

\subsection{Materials}

The printing substrate used in this research was the $W F C$ gloss fine art paper $300 \mathrm{~g} / \mathrm{m}^{2}$, which is commonly used in commercial printing and packaging. Optical characterization of the substrate was first performed by measuring the gloss value and colorimetric coordinates.

The black ink (SUNCHEMICAL SUNLIT EXPRESS PROCESS BLACK) was printed on the substrate by the means of the PRÜFBAU multipurpose printability tester MZ II. The PRÜFBAU multipurpose printability tester MZ II is a laboratory printability tester which enables printing in defined conditions with low amounts of printing materials.

The printing conditions were the ink amount $-0.15 \mathrm{~cm}^{3}$, the printing speed $-1 \mathrm{~m} / \mathrm{s}$ and the printing pressure -150 $\mathrm{N} / \mathrm{cm}^{2}$. The prints were in accordance with the ISO 12647$2: 2013$ norm $\left(\Delta E_{\mathrm{ab}} \leq 5\right)$. Ink was applied with a micro pipette into the distribution system of the unit (the rubber-metal roller system with rotational and axial movement) with the spread of 30 seconds in order to get an even ink film which would be transferred to the printing roller. Printing was performed after the substrate was conditioned for 24 hours in a room with a temperature of $21 \pm 1^{\circ} \mathrm{C}$ and relative humidity of $50 \pm 5 \%$, which is common in printing processes [8].

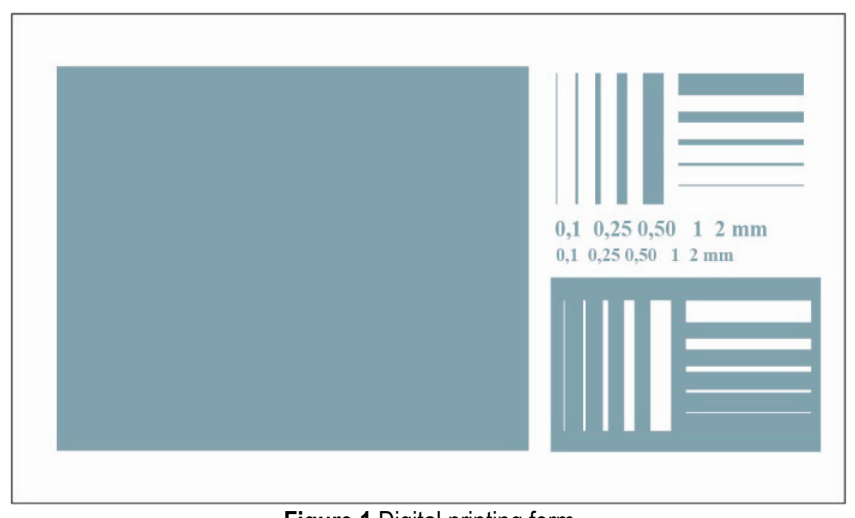

Figure 1 Digital printing form

The printed samples were then optically characterized by the measuring printing gloss and colorimetric coordinates. For the needs of the varnishing evaluation, the digitalized printing form is used, which includes a full tone patch and lines in positive and negative (Fig. 1). The digitalized printing form was before the varnishing process ripped in the surface coverage of $60 \%, 70 \%, 80 \%, 90 \%, 100 \%$. The $200 \%$ surface coverage was made as two overprints of the $100 \%$ printing form.

The digitalized printing forms (various surface coverage) were printed as an inkjet OPV by ROLAND VERSAUV LEC 300. The difference between the gloss and matte finish is in the UV drying technique, where to get the matte finish, UV drying receives a different energy than its gloss counterpart.

The matte finish requires two sets of UV LED lamps in which the first lamps cure the varnish, while the second lamps destroy the remaining cross-links in the polymer and create micro cracks in the surface. This is a common phenomenon in flexography plate making when the polymer is overexposed to the UV light. [4].

A total of 24 samples were prepared for this experiment. Samples were separated into four groups of six pieces denominated as WG (samples without black ink with a gloss finish), WM (samples without black ink with a matte finish), BG (samples with black ink and a gloss finish) and BM (samples with black ink and a matte finish). The samples in the group are denominated with the numbers $60-200$ that come from the surface coverage of the ripped digital printing form.

\section{MEASURING METHODS}

The evaluation of samples was performed by using optical methods and by visual evaluation of microscopic images.

\subsection{Optical Measurements of Paper, Prints and Varnished Samples}

In order to assess the colorimetric characteristics of the prepared samples, the CIE $L^{*} a^{*} b^{*}$ values were measured by the reflective spectrophotometer X-rite eXact. The used spectrophotometer is compliant to the ISO 5-4:2009 norm. Measurement conditions were $0 / 45^{\circ}$ geometry, $2^{\circ}$ standard observer, D50 illuminant and M1 measuring filter.

The results of measurements were transferred to the computer and the colour difference was calculated according to the $\Delta \mathrm{E}_{00}$ equation. Each sample was measured ten times. [10]

\subsection{Print Gloss Method}

The prepared samples, as well as the plain paper substrate (without varnishing or printed ink), were measured by using a glossmeter that measures the reflective glare in which light intensity is measured over a range of reflected angles. The intensity of the reflected light depends on the material and the angle used for illumination [11]. The remaining part of light that does not reflect is absorbed or diffuses per impact [12].

In this research, the Elcometer 407 glossmeter (measuring $60^{\circ}$ geometry) was used for the measurements ('Statistical Glossmeter Operating Instructions', n.d.). Each 
sample was measured ten times before UV varnishing and after it. Gloss was measured at the angle of $60^{\circ}$. [11, 13].

\subsection{Microscopic Analysis of the Elements}

A microscopic varnish element analysis was performed by means of the Leica DM 2500 microscope in order to assess the transfer of elements (positive and negative lines and text) that were printed in varnish with a gloss and matte finish. After taking the microscopic images at the enlargement of $25 \times$, they were visually assessed.

\section{$4 \quad$ RESULTS AND DISCUSSION}

Every measuring method was performed ten times after which the average values and standard deviation were calculated.

\subsection{CIE $L^{*} a^{*} b^{*}$ Coordinates and Colour Difference}

In Figs. $2-4$, one can see the colour coordinates of the evaluated samples. The dots in the diagrams are average values and the error bars represent the standard deviation of measurements.

One can observe that varnishing in both the gloss and matte finish acts as an overtop layer that makes the under image slightly darker (Fig. 2). With an increase in surface coverage, the values of the varnishing of the lightness do not substantially decrease.

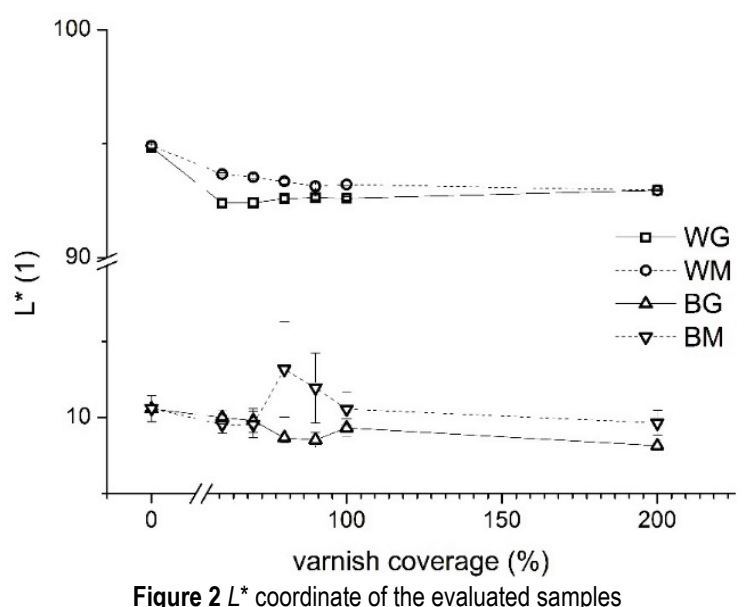

One can also see that the standard deviation of the printed samples (BM and $\mathrm{BG}$ ) is higher than the one of the paper samples (WG and WM). This behaviour is probably due to the high lightness of paper samples. Additionally, the highest deviation of the results is on the printed samples with a matte varnish finish (BM). This effect can be a result of light refraction due the micro surface roughness of the matte finish (Fig. 3) with the usage of the FM screening technique that was used in inkjet varnishing within this case $[14,15]$.

Moreover, varnish is applied via inkjet in the form of a droplet which, especially in the case of TV/surface coverage lower than $100 \%$, is shaped as a dome on the surface. This could lead to distortion in the reflected light [16] and therefore influence the measuring process, i.e. the measuring results.

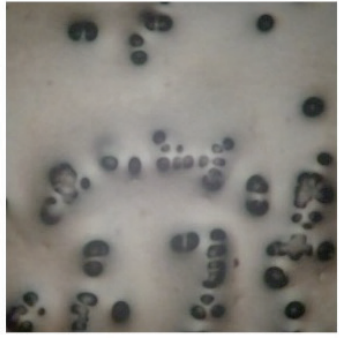

a)

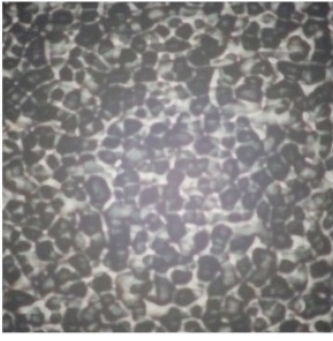

b)
Figure 3 Varnished surfaces printed with a $100 \%$ surface coverage value on a black solid with a gloss (a) and matte (b) finish enlargement of $25 x$.

In the case of $a^{*}$ and $b^{*}$ coordinates (Figs. 4 and 5), one can see that on the researched samples, varnishing causes a very small colour shift. Apart from the BG (a printed sample with a gloss finish varnish), all samples show the same pattern - the $b^{*}$ coordinate increases when applying varnish with a surface coverage value of $60 \%$ and the values then drop.
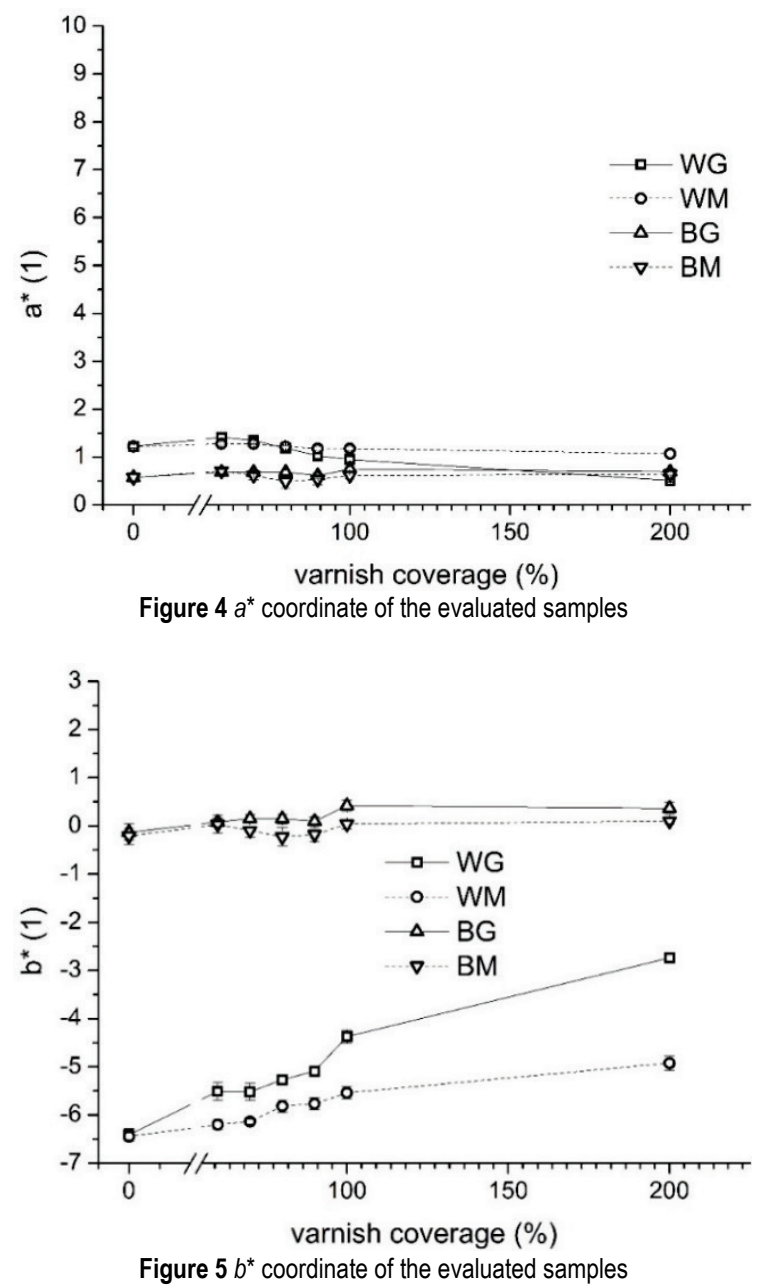

Although the paper and black print should be near the lightness axis ( $a^{*}$ and $b^{*}$ near 0$)$, it can be seen that there is a large difference between the paper and black print in the $b^{*}$ 
coordinate. Furthermore, opposite to the influence of the surface/tonal value of varnishing on the $a^{*}$ coordinate, adding varnish on the surface causes an increase of the $b^{*}$ coordinate on all researched samples (Figs. 4 and 5).

The varnish shifted the sample colour into the yellowish area, which is a common issue with all varnishing techniques. These results show that the usage of inkjet UV LED leads to the same issue and it cannot be avoided or decreased with lower surface coverage values.

Additionally, increasing the surface coverage of the varnish applied on the unprinted samples (WG and WM) causes a high increase of the $b^{*}$ coordinate. This could be due to the fact that varnish layers the filters and blocks the operation of optical brighteners [17].

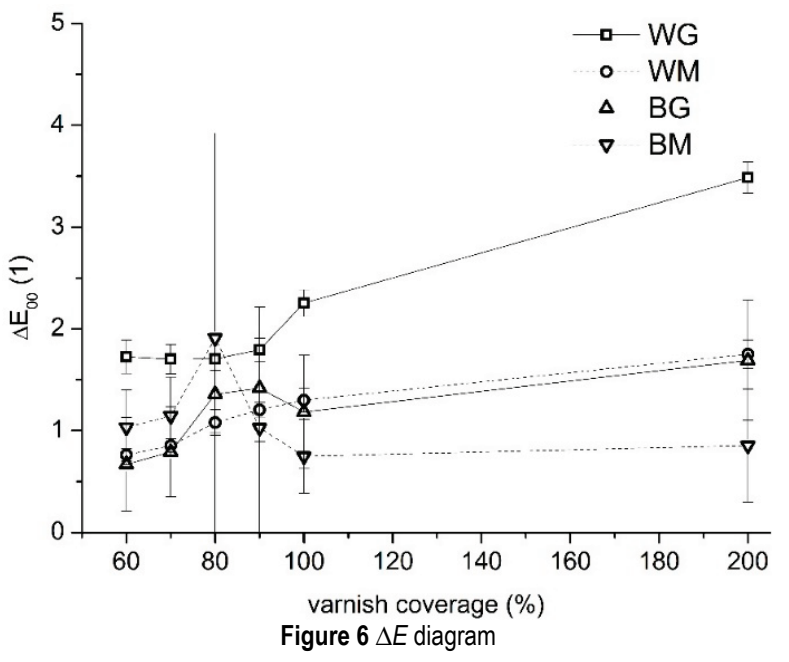

In the case of $\Delta E_{00}$, both the gloss and matte finished samples show the same trend. Generally, the colour difference increases with the increase of the surface coverage of the applied varnish (Fig. 6). Nevertheless, the calculated colour difference is on all samples (except the WG-200) under three, which is almost not visible by the human eye [10].

\subsection{Print Gloss}

Gloss enhancement or the matt finish effect, as described in the text above, are the most important visual reasons why varnishing is being done onto printed samples. As expected, gloss finished samples have a gloss value peak when varnishing with a surface coverage of $200 \%$. It can also be seen that on the black printed sample, the difference between varnishing with a surface coverage value of 100 or $200 \%$ results with nearly the same gloss value, which is why double varnish consumption is not affordable. A high difference between gloss on the BG-90 and BG-100 can be linked to the fact that at $100 \%$ TVI, the surface is fully covered with varnish and therefore higher light reflection is caused [14].

On the other hand, the matte effect causes the gloss value to drop significantly on the black printed sample (BM), as the pattern of the matte finish, which is visible in Fig. 3b, has more cracks than the ink film itself.
As opposed to the ink film, the paper substrate has certain irregularities on the surface, which at higher surface coverage values of varnishing ((90 and $100 \%)$ are mostly covered resulting with higher gloss value (Fig. 7). Doubling the varnish amount (varnishing with the tv/coverage of $200 \%$ ) could be justified in the case of the paper substrate as the gloss value is lower than on the unvarnished sample (however, covering the surface would mean protecting the surface), but would not be affordable for the black printed sample.

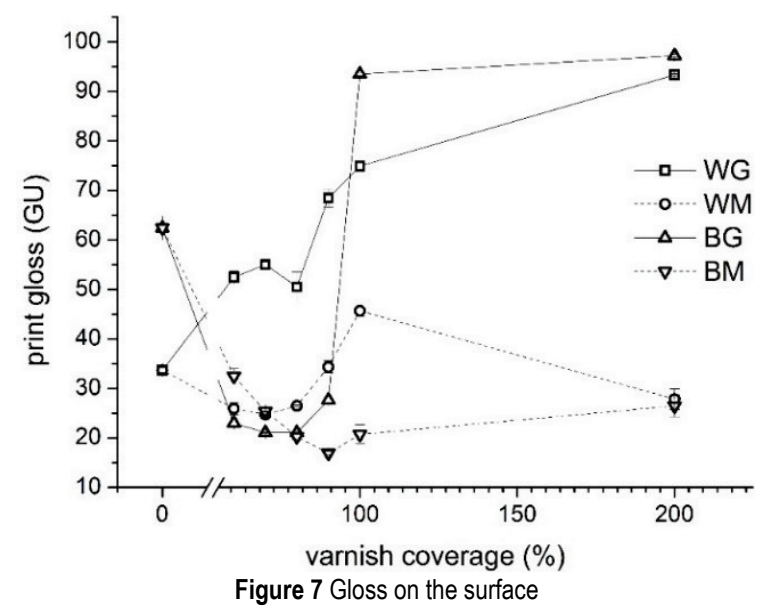

\subsection{Microscopic Varnish Elements' Analysis}

The analysis of the elements printed with varnish was performed on the numbers 0 and 1 of the $6 \mathrm{pt}$ font size and a positive line with the width of $0,25 \mathrm{~mm}$. These elements were chosen as they include elements with the areas in the direction of the printing head and other angles. The goal was to evaluate the surface appearance of the small elements and their applicability in the pattern varnishing with a smaller surface coverage and a surface finish (gloss or matte). The microscopic images are presented in Fig. 8, where numbers by rows denote the surface coverage value of varnishing, while the letters under columns denote a sample group.

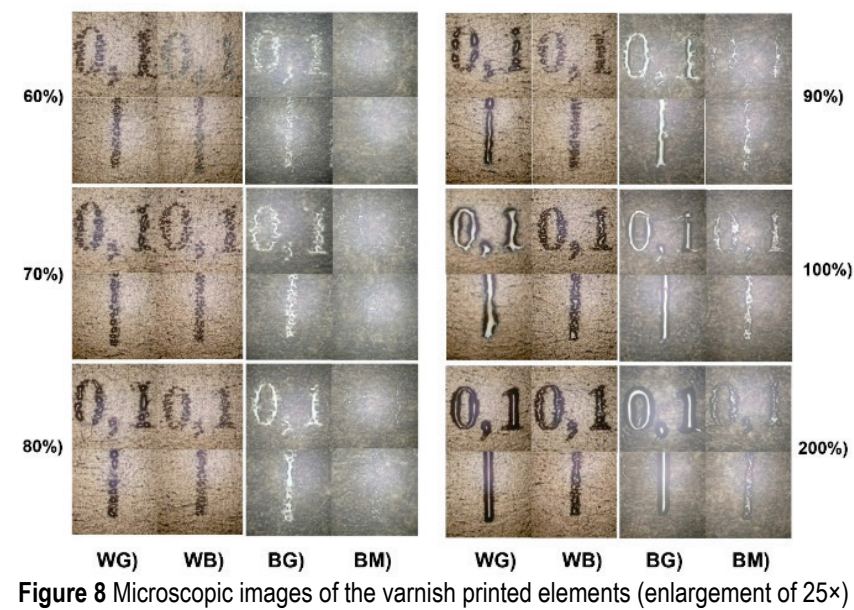


One can see that on the gloss finished samples, both on the paper substrate and black printed substrate, varnish printed elements are clearer to see than on the matte ones (Fig. 7). Moreover, it can be seen that on the black printed samples, varnish elements with a matte finish are not even visible if varnishing is conducted with a surface coverage under $90 \%$. The gloss finish reflects the light in a way that creates a natural shadow which visually appears as an outline around the varnish printed element, creating a contrast which will differentiate the elements from the surface. In matte finish samples, the varnished area surface has a bigger amount of micro surface roughness that significantly distorts the light $[4,9,14]$.

Assessing the printed pattern, one could clearly recognize the varnish printed elements on the printing substrate and the black printed sample when varnishing with a $60 \%$ surface coverage, but the effect of the gloss is noticeable when varnishing with at least a $90 \%$ surface coverage.

On the other hand, the varnish printed elements with a matte finish are recognizable by $70 \%$ varnishing on paper and $100 \%$ varnishing on the black printed substrate. Varnishing with $200 \%$ decreases the glossy effect on the paper substrate, while it enables clearer varnish printed elements on the black printed substrate.

\section{CONCLUSIONS}

This research was conducted in order to research the usability of the UV LED inkjet varnishing in adding value to the prints produced in a conventional printing technique (offset). The prepared samples were optically characterized to see if there are any optical differences caused by the varnishing process. Furthermore, the evaluation of the different amounts of varnish and the application of pattern varnishing was also performed.

The results showed that the UV LED varnishing causes small colour differences which are in most cases not visible to the human eye. On the other hand, gloss measurements showed that varnishing with a gloss finish significantly increases gloss values on the substrate and the printed ink film.

Regarding pattern printing, it could be concluded that the use of lower amounts of varnish by applying varnish as a FM screen in coverage/tonal values lower than $100 \%$ will result with good results in the matte finish varnishing. On the other hand, if the print has to have a high gloss value due to the screening pattern, one must varnish in a $100 \%$ coverage value, but the double amount (a surface coverage of $200 \%$ ) is not affordable as it increases the print's gloss value by a few GU.

Additionally, it has been noticed that the handling of samples that were to be varnished caused some problems as any contamination of the surface, such as the ones from hand grease, creates patterns on the varnish film that are visible.

To conclude, varnishing with the UV LED technology would not be recommended as an offline process, but it could be useful as an inline hybrid technology as it offers more variations in varnishing processes (varnish amount, varnishing pattern) in comparison to conventional technologies.

\section{REFERENCES}

[1] Kipphan, H. (2001). Handbook of Print Media. Berlin, Germany: Springer-Verlag Berlin, 45-60. https://doi.org/10.1007/978-3-540-29900-4

[2] Smyth, S. \& Smithers, P. (2017, April 13). Digital Printing to Continue to Take Market Share from Offset Presses. Retrived from https://www.inkworldmagazine.com/issues/2017-0501/view_experts-opinion/digital-printing-to-continue-to-takemarket-share-from-offset-presses/

[3] Digital Printing Market Size, Share, Trends, Opportunities And Forecast. (2018, April) Retrived from https://www.verifiedmarketresearch.com/product/digitalprinting-market/.

[4] Majnaric, I., Bolanca Mirkovic, I., Birta, A., \& Mustac, S. (2013). The Influence of the Extreme Thick Applied Layers of Varnish on Color Properties of LED UV curing inkjet prints. AIC Color 2013, 1269-1272.

[5] Dolic, J., Pibernik, J., \& Majnaric, J. (2014). Influence of UV Varnish pattern effect on Print Quality. J. Imaging Tecnol., 58(6), 060501-060509. https://doi.org/10.2352/J.ImagingSci.Technol.2014.58.6.060501

[6] Yang, L. (2003). Ink-Paper Interaction: A study in ink-jet color reproduction. Dissertation, Linkoping Studies in Science and Technology, no. 806, 21-28.

[7] Magdassi, S. (2010). The Chemistry of INK jet inks. Singapore, World Scientific Publishing Co., 161-177.

[8] Krizmanic, K., (2010). Kvalitativne Karakteristike digitalnog i konvencionalnog ofsetnog tiska. Master thesis, University of Zagreb, Faculty of Graphic Arts, 7-12. (in Croatian)

[9] Majnaric, I., Golubovic, K., \& Bolanca Mirkovic, I. (2012). New methods of varnishing and their influence on optical properties of cardboard packaging. Matrib 2012 Book of abstracts, Vela Luka, Croatia, 179-189.

[10] Sharma, G., Wu, W., \& Dalal, M. (2005). The CIEDE2000 color-difference formula: Implementation notes, supplementary test data, and mathematical observations. Color Res. Appl., 30(1), 21-30. https://doi.org/10.1002/col.20070

[11] Nadal, M. \& Thompson, A., (2000). New Primary Standard for Specular Gloss Measurements. Journal of Coatings Tecnology, 72(991), 61-66. https://doi.org/10.1007/BF02720526

[12] Hunter, R. S. (1934). Methods of determining gloss. J. Res. Natl. Bur. Stand., 30(1), 28-29.

[13] Elcometer Lim. (2009). Statistical Glossmeter Operating Instructions, instruction manual 406L.

[14] Karlović, I. \& Novaković, D. (2011). Effect of Different Coating Amounts on the Surface Roughness and Print Gloss of Screen Coated Offset Prints. J. Imaging Sci. Technol., 55(2), p. 020501. https://doi.org/10.2352/J.ImagingSci.Technol.2011.55.2.020501

[15] Car, I., Majnaric, I., \& Lozo, B. (2018). Colorimetric Changes Caused by UV Varnishing. In CIGT 2018, 37-40.

[16] Macinic, D. (2011). Utjecaj efekta lakiranja na optička svojstva otisaka. Master thesis, University of Zagreb, Faculty of Graphic Arts. (in Croatian)

[17] Majnaric, I., Bolanca Mirkovic, I., \& Golubovic, K. (2012). Influence of UV curing varnish coating on surface properties of paper. Technical Gazette, 19(1), 51-56. 


\section{Authors' contacts:}

Tomislav Hudika, mag. ing., Research Assistant

(Corresponding author)

Department of Graphic Materials and Printing Forms,

Faculty of Graphic Arts, University of Zagreb,

Getaldićeva 2, 10000 Zagreb, Croatia

Tel: +385 (0)1 2371080

thudika@grf.hr

Igor Majnarić, PhD, Associate Professor

Department of Printing Processes,

Faculty of Graphic Arts,

University of Zagreb,

Getaldićeva 2, 10000 Zagreb, Croatia

Tel: +385 (0)1 2371080

imajnaric@grf.hr

Tomislav Cigula, PhD, Assistant Professor

Department of Graphic Materials and Printing Forms,

Faculty of Graphic Arts, University of Zagreb,

Getaldićeva 2, 10000 Zagreb, Croatia

Tel: +385 (0)1 2371080

tcigula@grf.hr 